\title{
LETTERS
}

\section{Neurosyphilis is an easily missed cause of encephalopathy}

I enjoyed reading Dr. Budhram and colleagues' case report on neurosyphilis mimicking autoimmune encephalitis. ${ }^{1}$ I thank the authors for bringing attention to this important (albeit uncommon) presentation, which can be easily missed or forgotten unless it is ingrained in our minds as part of the workup for these types of subacute encephalopathic presentations. For example, as part of Neurology's educational outreach efforts, we recently surveyed neurologists and residents on the differential diagnosis of a real-life case of a 64-year-old woman with neurosyphilis who similarly presented with subacute encephalopathy (but no seizures) and had bilateral mesial temporal lobe hyperintensities. ${ }^{2}$ Nearly half of the respondents recognized the importance of considering paraneoplastic and autoimmune causes in this presentation and a third recognized herpes encephalitis as another potential cause, but only $14 \%$ considered neurosyphilis in their differential diagnosis or workup. In the absence of reliable data, it is difficult to know the extent to which neurosyphilis is missed in clinical practice, but case reports like that by Budhram and colleagues in general medical journals such as CMAJ will hopefully serve as a reminder to both general and specialist clinicians to incorporate this curable condition into their standard workup for encephalopathy.

\section{Aravind Ganesh MD}

Neurology Resident, Department of Clinical Neurosciences, University of Calgary, Calgary, Alta.; Clinical Research Fellow, Centre for Prevention of Stroke and Dementia, Nuffield Department of Clinical Neurosciences, University of Oxford,

Oxford, UK

- Cite as: CMAJ 2017 November 13;189:

E1401. doi: 10.1503/cmaj.733354

\section{References}

1. Budhram A, Silverman M, Burneo JG. Neurosyphilis mimicking autoimmune encephalitis in a 52-year-old man. CMAJ 2017;189:E962-5.

2. Bhai S, Biffi A, Bakhadirov K, et al. Mystery Case: a 64-year-old woman with subacute encephalopathy. Neurology 2015;85:e64-5.

Competing interests: Aravind Ganesh is on the editorial board of Neurology's Residents and Fellows section. 